\title{
Gall bladder carcinoma in a young male - a rare presentation
}

\author{
${ }^{1}$ Poorna Chandra Thejeswi, ${ }^{2}$ Guru Prasad Reddy, ${ }^{3}$ Shivananda prabhu \\ ${ }^{1,2,3}$ (Department of General Surgery, Kasturba Medical College, Mangalore, Manipal University, India)
}

\begin{abstract}
Gallbladder carcinoma in a setting of acute cholecystitis is very rare occurrence in young patients (younger than 30 years).Moreover a definitive preoperative diagnosis is difficult. The purpose of this report is to present an unusual case of gallbladder carcinoma in a patient who presented with acute cholecystitis. A 27 year old male presented to the casualty with pain in the right hypochondriacregion for which he was admitted and evaluated and was diagnosed as suffering from acute calculous cholecystitis. Laparoscopic cholecystectomy was done and the histopathology was suggestive of gallbladder adenocarcinoma.

Conclusion: Carcinoma of the gallbladder is an aggressive malignancy which occurs predominantly in elderly people. Besides the exceptional cases detected incidentally at the time of cholecystectomy for gallstone disease, which are usually early stage, the prognosis for most patients is poor. Unfortunately, many of these tumors are unresectable at presentation, and most must be managed nonoperatively.Gallbladder carcinoma is a rare occurrence and is to be suspected in patients with complaints of cholelithiasis for long duration and histopathological examination of the cholecystectomy specimens has to be routinely carried out.
\end{abstract}

Keywords: Carcinoma, Cholecystitis, Gallbladder, Young

\section{Introduction}

A 27 year old male patient presented to the casualty with complaints of pain in the right hypochondrium from one day,pain was dull aching in type, non-radiating,associated with non-bilious vomitings, no history of fever, diarrhoea or urinary disturbances could be elicited.

On clinical examination, there was no pallor or icterus and vitals were normal. Patient had tenderness in the right hypochondriac region,Murphy's sign was positive.There was no guarding or rigidity,and no lump was palpable.

The following differential diagnoses were considerd - acute cholecystitis, pancreatitis,peptic ulcer disease,acute hepatitis,liver abscess and patient was admitted and evaluated in the form of blood investigations and imaging modalities.total bilirubin 0.4,direct bilirubin 0.14,AST 39,ALT 51,ALP 226,CRP 38(positive),serum amylase 60,lipase 26,LDH 299, GGT 126,CA19.9 6.35,CEA 1.94,serum creatinine 1,bleeding time 2 min,clotting time 3 min,prothrombin time 18.7 and INR 1.27,haemoglobin 12.9,total counts 10,500(neutrophils 76,eosinophils 6.5),platelets 2.15lakhs.

CECT showed enhancing lesions in the fundus of distended gall bladder and in mid common bile ductneeds evaluation,cholecystolithiasis,enlarged appendix with appendicolioth within and bilateral non obstructive renal calculi.

ERCP with biliary sphincterotomy and common bile duct stone extraction was done. MRCP showed intraluminal/extrinsic nodular lesion measuring approximately 1.8 to $2 \mathrm{cms}$ noted in the proximal intrahepatic $\mathrm{CBD} /$ indenting lumen causing proximal CHD and intrahepatic biliary dilatation.Small distal CBD filling defects/o calculus $6 \mathrm{~mm}$. Gall bladder shows polypoid fundal mucosal lesion measuring 1.5 to $2 \mathrm{cms}$ approximately with cholecystolithiasis.Calculus impacted at the neck of gall bladder measuring $18 \mathrm{~mm}$. Possibility of fibrovascular/hyperplastic polyps in the GB and CBD Intrahepatic segment/extrinsic nodular lesion indenting CBD to be considered with distal CBD calculus $6 \mathrm{~mm}$

Intraoperatively enlarged lymphnode of Lund was noted and multiple calculi were present in the gallbladder(arrow in Fig 1). 


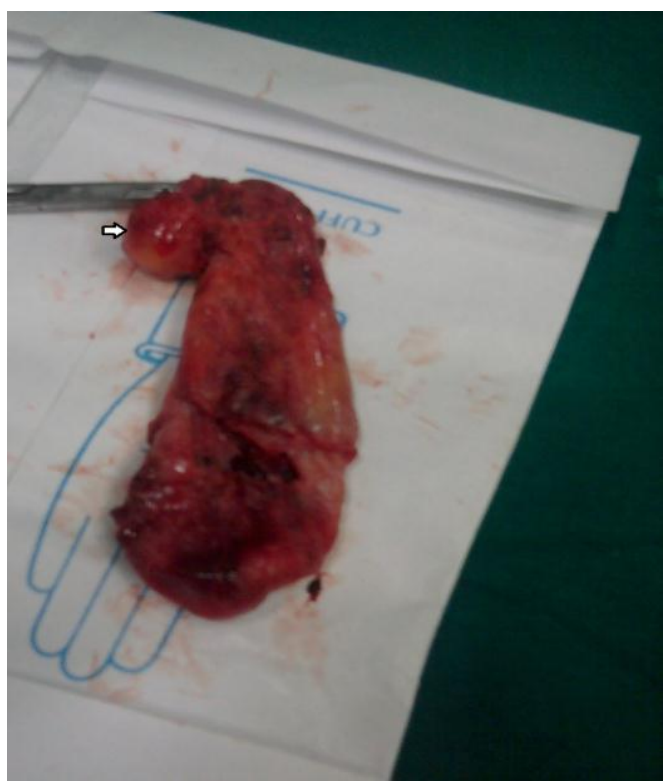

Fig 1.Gross specimen showing enlarged lymph node of Lund

Post procedure MRCP - decompression of biliary tract noted with no calculus in distal CBD.Calculus impacted at neck of GB noted with multiple intraluminal calculi and polypoidal lesion persisting in the gall bladder fundus as in prior scan. The nodular lesion adjacent to the proximal CBD is 61 showing exrinsic indentation and displacement of CBD-s/o ?lymph node/nodular lesion in liver parenchyma. Histopathology impression was sugggestive of differentiated adenocarcinoma of gall bladder.TNM stage II B (T2 N1 Mx).

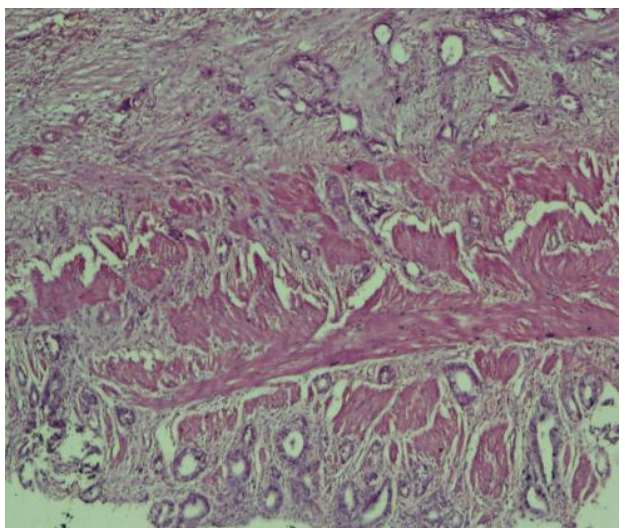

Fig 2. Section from wall of gallbladder showing multiple irregular glands infiltrating lamina propria upto serosa with marked desmoplastic stroma and areas of haemorrhage and necrosis.

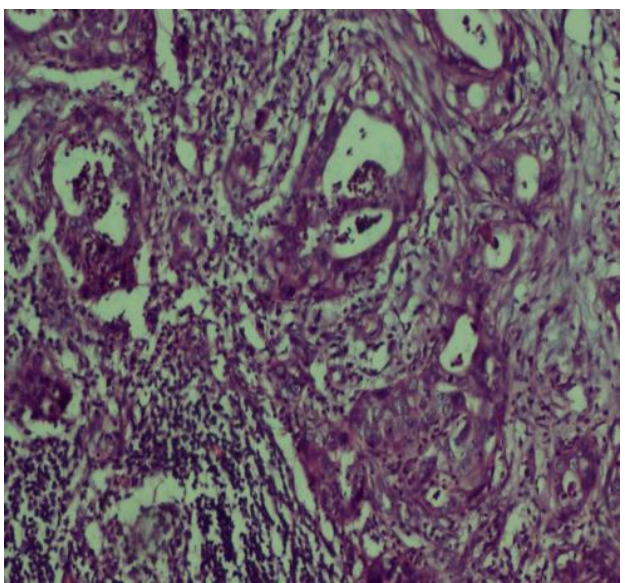

Fig 3. Thickened wall of gallbladder with papillary projections lined by dysplastic cuboidal to columnar epithelial cells. 
Patient was advised extended hepatectomy but was not willing.

Medical oncologist opinion was sought and was advised chemotherapy with Gemcitabine.Patient received 6 cycles of chemotherapy and post chemotherapy evaluation revealed no residual disease.

\section{Discussion}

Gallbladder cancer is the fifth most common gastrointestinal malignancy, with about five thousand new cases diagnosed yearly in the United States. Cancer of the gallbladder is two to three times commoner in women compared to men, due the higher incidence of gallstones in women. More than three fourths of patients with this malignancy are above 65 years ${ }^{1 .}$ The pathogenesis is likely related to chronic inflammation. Gallstones are the most common factor because of the high prevalence in the general population.

Gallbladder cancer is seven times commoner in presence of cholelithiasis and chronic cholecystitis.In addition, the risk for developing gallbladder cancer is higher in patients with symptomatic gallstones than in patients with asymptomatic gallstones. About $1 \%$ of elective cholecystectomies done for cholelithiasis have an occult gallbladder cancer. $90 \%$ of cancers of the gallbladder are classified as adenocarcinoma. Squamous cell, oat cell and adenosquamous cancers and carcinoid tumors are much less frequent. $6 \%$ of gallbladder adenocarcinomas demonstrate papillary features histopathologically; these tumors are commonly diagnosed while localized to the gallbladder and are also associated with an improved overall survival. At diagnosis, 25\% of cancers are localized to the gallbladder wall, $35 \%$ have associated metastases to regional lymph nodes or extension into adjacent organs, and $40 \%$ have already metastasized to distant sites.

Gallbladder cancer most commonly presents with right upper quadrant pain often imitating cholecystitis and cholelithiasis. Weight loss, jaundice, and an abdominal mass may also be the presenting symptoms. About $40 \%$ of patients present with symptoms of chronic cholecystitis. Another presentation is similar to acute cholecystitis, with a short duration associated with vomiting, fever, and tenderness. Gallbladder cancer is often misdiagnosed as chronic cholecystitis, pancreatic cancer, acute cholecystitis, choledocholithiasis.

A rational approach for gallbladder carcinoma by Glenn and Hays ${ }^{3}$ in 1954 included wedge resection of the gallbladder bed and regional lymphadenectomy. Definitive resection depends on the stage and location of the primary as well as whether it is a repeat resection after a previous cholecystectomy. Simple cholecystectomy can be done for T1 (stage Ia) tumors. Any suspicious nodes should be removed for pathologic examination. Stage Ib, II, and selected stage III $(\mathrm{T} 4, \mathrm{~N} 0)$ tumors should be treated with resection of the gallbladder along with liver segments IVb and V, and lymph node dissection. Stage IV tumors should be treated with palliation ${ }^{3}$.

The goal is to achieve a negative margin, encompassing the infiltration into the liver. Sometimes,after prior cholecystectomy or biliary operations, it is difficult to differentiate between scar and tumour in porta hepatis ${ }^{3,4}$.

Patients with metastases beyond hepatoduodenal ligament have poorer prognosis, and palliation is usually advised. Some investigators reported cases of long term cure in patients with distant nodal metastasis with radical surgery ${ }^{5}$,but most reported a poor outcome, which does not favour the morbidity of the extended resection.

Chemotherapy has been used for palliation of unresectable tumor. Trials have clubbed gallbladder and bile duct cancers together. An analysis of 103 trials in biliary tree cancer suggests that gall bladder cancer may have a higher response to chemotherapy, but with lower survival compared to bile duct cancers. ${ }^{6,7}$ Chemotherapy has been used extensively in advanced gallbladder cancer and gemcitabine-based combination (with cisplatin and oxaliplatin or with capecitabine) show longer survival with than with gemcitabine alone ${ }^{7}$.

\section{Conclusion}

Gallbladder carcinoma in younger age group though is a rare occurrence is to be suspected in patients with complaints of cholelithiasis for long duration and histopathological examination of the cholecystectomy specimens has to be routinely carried out.

\section{Abbreviations}

GB - Gallbladder, CBD _ Common bile duct, CHD - Common Hepatic Duct, ERCP - Endoscopic Retrograde Cholangiopancreaticography, MRCP - Magnetic Resonance Cholangiopancreaticography,ALP Alkaline phosphatase, AST - Aspartate transaminase, ALT - Alanine transaminase

\section{References}

[1]. Wistuba II, Gazdar AF. Gallbladder cancer: lessons from a rare tumour. Nature

[2]. Rev Cancer 2004;4:695 [PubMed: 15343276]

[3]. Misra S, Chaturvedi A, Misra NC, et al. Carcinoma of the gallbladder. Lancet

[4]. Oncol 2003;4:167 [PubMed: 12623362] 
[5]. Glenn F, Hays DM. The scope of radical surgery in the treatment of malignant tumors of the extrahepatic biliary tract. Surg Gynecol Obstet 1954;99:529.

[6]. 4.Bartlett DL, Fong Y, Fortner JG, et al. Long-term results after resection for gallbladder cancer. Implications for staging and management. Ann Surg 1996;224:639.

[7]. Shirai Y, Yoshida K, Tsukada K, et al. Radical surgery for gallbladder carcinoma. Long-term results. Ann Surg 1992;216:565.

[8]. Eckel F, Schmid RM. Chemotherapy in advanced biliary tract carcinoma: a pooled analysis of clinical trials. Br J Cancer 2007:96:896.

[9]. 7.Zhu AX, Hezel AF. Development of molecularly targeted therapies in biliary tract cancers: reassessing the challenges and opportunities. Hepatology 2011; 53: 695-704. 